\title{
Horologium mercatus : bene diagnoscitur, bene curatur ${ }^{1}$
}

\author{
André Boivin \\ Université du Québec à Chicoutimi
}

\section{Introduction}

Bon an mal an, une moyenne de 10000 faillites commerciales sont enregistrées au Canada et elles représentent des milliards de dollars de perte pour l'économie du pays. Pis encore, les choses ne s'améliorent pas avec le temps ${ }^{2}$. Mais toutes ces banqueroutes étaient-elles vraiment inévitables? N'aurait-t-on pas lancé la serviette un peu trop tôt ? Avant de liquider les actifs, a-t-on envisagé le prêt de dépannage ou encore la fusion? Nous croyons que trop d'entreprises sont euthanasiées à la suite d'un mauvais diagnostic. Voilà pourquoi nous proposons un outil de dépistage simple et efficace auquel nous avons ironiquement donné le nom scientifique d'Horologium mercatus et qui donne véritablement l'heure juste sur la viabilité future d'une organisation ${ }^{3}$.

\section{Bilan comptable ou bilan économique?}

À l'instar du bilan comptable, le bilan économique fait état de la valeur des actifs et des passifs à une date fixe. Mais les comptables et les économistes ne définissent pas la valeur de la même façon. Alors que les premiers l'établissent unilatéralement à partir de règles théoriques bien précises ${ }^{4}$, les seconds scrutent les marchés afin d'obtenir une valeur consensuelle dont ils ne sont ultimement que les messagers. Forcément, l'image que montrent comptables et économistes n'est pas du tout la même. Toute comparaison étant imparfaite, nous oserons quand même associer l'image comptable à une peinture par numéros, et l'autre à une photographie couleur. La première, quoique souvent jolie, ne rend pas toujours justice à l'original. La photographie, elle, ne peut mentir, car elle n'est que la réplique exacte d'un sujet réel. Voilà sans doute pourquoi, par exemple, on retrouve des photographies plutôt que des croquis sur les cartes d'identité.

Bien qu'il ne soit pas notre intention de faire ici le procès de la comptabilité, nous voulions quand même en souligner une certaine inconsistance. Lorsque les dirigeants d'une organisation sont confrontés à des choix stratégiques, ceux-là même qui lui assureront sa place au soleil dans un marché compétitif, il nous apparaît logique d'utiliser des mesures marchandes plutôt que des mesures comptables.

Cela étant, le bilan économique nous apparaît le seul choix rationnel. Fidèles à nous-mêmes, nous le baptiserons affectueusement Bonam mercatus $I^{5}$.

\section{Bonam mercatus I illustré}

Le tableau 1 illustre le Bonam mercatus I d'une organisation fictive à qui nous donnerons le nom d'ABC. À l'exemple du bilan comptable, ses deux côtés sont parfaitement balancés. Les éléments qui composent l'actif sont la valeur marchande des actifs tangibles (VMT) et la valeur marchande de l'achalandage (VMA) ${ }^{6}$.

Du côté des créances, on retrouve la valeur marchande des dettes (VMD) et la valeur économique nette espérée (VEN) des propriétaires ${ }^{7}$. Cette dernière est la pièce maîtresse d'Horologium mercatus. 
Tableau 1 - Bonam mercatus I de l'organisation ABC

\begin{tabular}{c|c|c|c|}
\hline \multicolumn{1}{|c|}{ ACTIF } & & PASSIF + AVOIR \\
\hline VMT & 15 & VMD & 12 \\
VMA & 2 & VEN & 5 \\
TOTAL & $\mathbf{1 7}$ & TOTAL & $\mathbf{1 7}$ \\
\hline
\end{tabular}

\section{Première transmutation : Bonam mercatus I devient Bonam mercatus II}

Avant de se métamorphoser en Horologium mercatus, Bonam mercatus $I$ doit subir une première transformation ayant pour but d'isoler la valeur économique nette espérée. Pour ce faire, on soustrait la valeur marchande des dettes de la valeur marchande des actifs tangibles (VMT - VMD), obtenant ainsi une valeur de liquidation (VL). De fait, cette dernière correspond au montant net dont disposerait l'organisation si elle remboursait l'ensemble de ses dettes avec l'argent récolté lors de la vente publique de ses actifs tangibles. Dans le cas d' $\mathrm{ABC}$, ce montant est égal à $15 \$-12 \$=3 \$$ (voir le tableau 2 ).

Après cette première transformation, Bonam mercatus $I$ se simplifie pour devenir Bonam mercatus II, tel qu'illustré au tableau 2. Comme son précurseur, il est parfaitement équilibré mais amputé de toutes ses dettes et d'une partie égale de ses éléments d'actif. Bref, Bonam mercatus II est un bilan de liquidation simulée ${ }^{8}$ dont la principale vertu est d'isoler la valeur économique nette espérée, véritable catalyseur de la mutation définitive vers Horologium mercatus.

Tableau 2 - Bonam mercatus II de l'organisation $\mathrm{ABC}$ après la liquidation des actifs tangibles et le remboursement de toutes les dettes

\begin{tabular}{|c|c|c|c|}
\hline \multicolumn{2}{|c|}{ ACTIF } & \multicolumn{2}{|c|}{ PASSIF + AVOIR } \\
\hline VL & 3 & VEN & 5 \\
\hline VMA & 2 & & \\
\hline TOTAL & 5 & TOTAL & 5 \\
\hline
\end{tabular}

\section{Horologium mercatus se dévoile enfin !}

À partir des valeurs contenues dans Bonam mercatus II, il est maintenant possible de fabriquer Horologium mercatus (voir la figure 1). De fait, cette dernière est un plan cartésien prenant la forme d'une horloge et dont l'abscisse et l'ordonnée représentent respectivement la valeur marchande de l'achalandage et la valeur de liquidation. Le point de rencontre des deux axes, le point d'origine, correspond à la valeur zéro (0) et les coordonnées cartésiennes (VMA, VL) représentent, elles, le lieux géométrique de la valeur économique nette espérée. Ultimement, c'est le quadrant à l'intérieur duquel cette dernière se retrouve qui permettra de poser un diagnostic sur la situation financière de l'organisation et de proposer, dans le cas où la VEN se situerait entre 3 heures et 12 heures, une méthode de réorganisation financière optimale lui évitant peut-être une injustifiable mise à mort.

Figure 1 - Horologium mercatus de l'organisation ABC

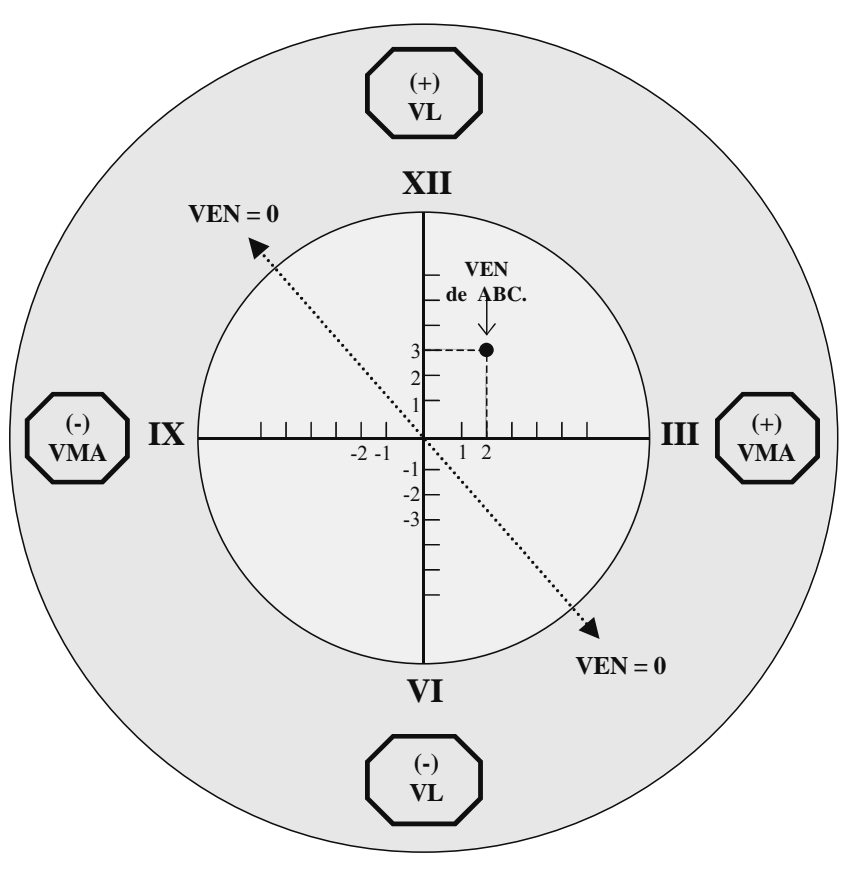

\section{Dis-moi quelle est ton heure et je te dirai qui tu seras}

La figure 2 illustre les cycles d'Horologium mercatus. À chacun d'eux correspondent un diagnostic et une solution appropriés que nous expliquons ci-après. 


\section{Entre 0 h 00 et 3 h 00}

Ce premier quadrant d'Horologium mercatus est porteur de bonnes nouvelles puisque tout y est positif (VL, VMA et VEN). C'est véritablement le royaume de la valeur ajoutée. D'une part, le risque de faillite est inexistant puisque la valeur de liquidation est plus grande que zéro. D'autre part, et surtout, les propriétaires ont tout intérêt à persévérer puisque l'exploitation leur permettra de s'enrichir, tel que l'indique la valeur marchande positive de l'achalandage. La valeur économique nette des propriétaires étant égale à la somme de ces deux dernières $(\mathrm{VEN}=\mathrm{VL}+$ VMA), il va sans dire que plus elles seront grandes, plus la valeur économique nette espérée le sera. Voilà sans doute un contexte d'exploitation idéal.

Figure 2 - Les cycles d'Horologium mercatus

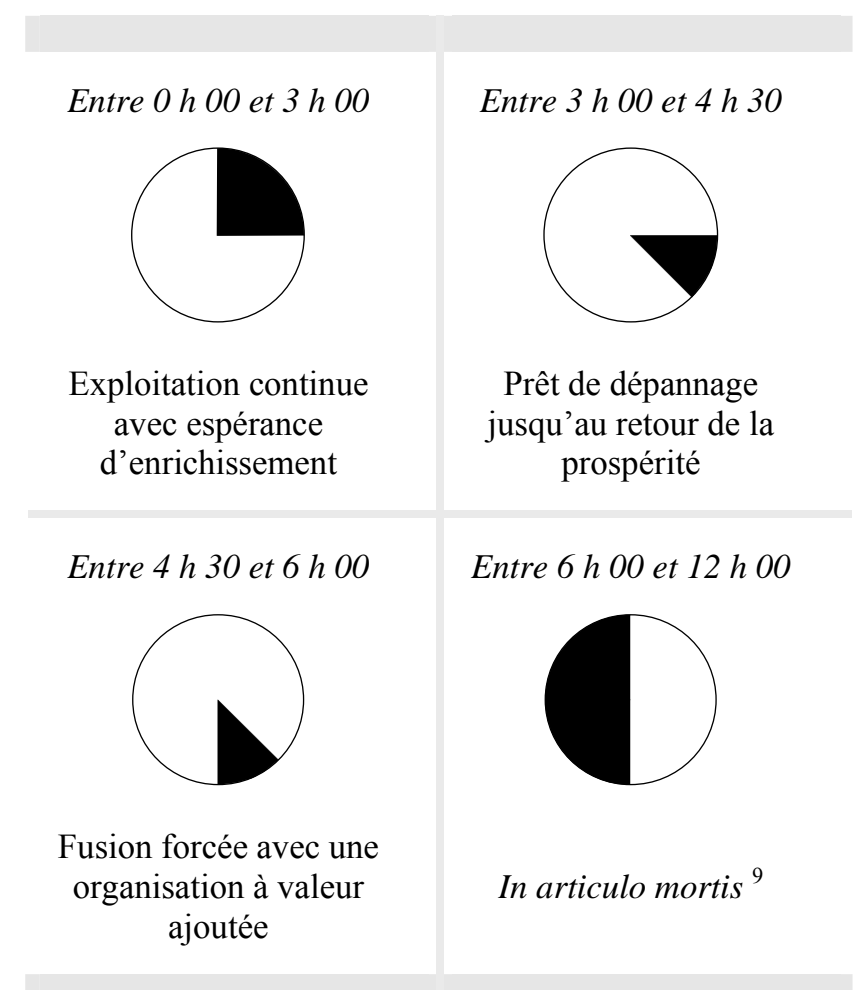

\section{Entre 3 h 00 et 4 h 30}

Cette première partie du deuxième quadrant ${ }^{10}$ n'est pas une zone de navigation de tout repos. Les eaux y sont plutôt troubles puisque la valeur de liquidation est négative. En pareil cas, l'organisation est techniquement en situation de faillite, car la liquidation des actifs tangibles ne suffirait pas à rembourser la totalité des dettes $(\mathrm{VL}<0)$. Bonne nouvelle toutefois, l'enri- chissement espéré tel que mesuré par la VMA est positif et suffisamment élevé pour conserver aux propriétaires une valeur économique nette espérée positive $($ VEN $>0)$. Ils auraient donc tout à perdre si on liquidait l'organisation puisqu'ils ne pourraient pas toucher l'enrichissement escompté. Ce dont ils ont besoin, en fait, c'est d'un sursis, juste le temps que la rentabilité revienne. En pareil cas, le prêt de dépannage semble la solution optimale.

\section{Entre 4 h 30 et 6 h 30}

Si la valeur économique nette espérée se situe dans cette deuxième moitié du deuxième quadrant, il y a alors péril en la demeure. Comme la valeur de liquidation est inférieure à zéro, l'organisation est techniquement en situation de faillite. De surcroît, la valeur marchande de l'achalandage, bien que positive, ne suffit pas à annuler la valeur de liquidation négative. $\mathrm{Au}$ net, donc, la valeur économique tombe sous zéro, ce qui signifie que les propriétaires sont pratiquement ruinés et qu'ils n'ont plus rien à perdre. Si on les laissait diriger l'organisation, ils seraient alors tentés de refuser des projets dont la valeur ajoutée ne profiterait qu'aux créanciers ${ }^{11}$. Conséquemment, il faut leur retirer le contrôle de leur organisation. Mais n'oublions pas que la valeur marchande de l'achalandage est positive. En fusionnant l'organisation avec une autre et en laissant le soin à de nouveaux propriétaires (dont la VEN est positive) de gérer la nouvelle entité, l'enrichissement net que représente la VMA pourra ainsi se concrétiser.

\section{Entre 6 h 00 et 12 h 00}

L'organisation dont la VEN se situe à l'intérieur du troisième et du quatrième quadrants d'Horologium Mercatus est à l'article de la mort économique. La valeur marchande de l'achalandage y est négative, ce qui implique que la poursuite de l'exploitation appauvrirait les propriétaires. C'est vraiment le pire des scénarios possibles, et la solution qui s'y rattache est dramatique : la liquidation immédiate des actifs et l'abandon des affaires. En effet, à quoi bon continuer d'exploiter un commerce si aucune valeur ajoutée n'est possible?

\section{Conclusion}

Horologium mercatus est un outil de diagnostic ${ }^{12}$ fort attrayant, relativement facile d'usage et dont la princi- 
pale qualité est d'anticiper le futur probable d'une organisation, au sens large du terme. Grâce à elle, on pourrait, par exemple, donner l'heure juste sur la viabilité économique d'une entreprise, d'une ville, voire d'une région toute entière. Dans quel quadrant d'Horologium mercatus Saguenay (ville ou région) se situe-t-elle ? Notre région est-elle réellement à 1'article de la mort, comme plusieurs le prétendent? Par rapport aux autres municipalités du Québec ou d'ailleurs, comment Saguenay performe-t-elle ? Si, par hasard, l'heure économique de Saguenay se situait entre 3 et 6 heures, quelles actions faudrait-il alors entreprendre pour remonter l'horloge (entre 0 et 3 heures évidemment) et lui permettre ainsi de renouer avec la prospérité ? Les fusions municipales étaient-elles vraiment toutes appropriées ? Voilà certes d'épineuses questions auxquelles Horologium mercatus aurait pu contribuer à répondre.

\section{Horologium mercatus devrait être produit annuellement, en même temps que les états financiers vérifiés, permettant ainsi de mettre simultanément en lumière l'avenir probable et l'histoire récente de l'organisation.}

À notre humble avis, Horologium mercatus devrait être produit annuellement, en même temps que les états financiers vérifiés, permettant ainsi de mettre simultanément en lumière l'avenir probable et l'histoire récente de l'organisation. Pourquoi, par exemple, ne pas demander à l'expert en évaluation d'entreprise de produire annuellement Horologium mercatus pour le remettre ensuite à l'expert-comptable qui l'ajouterait à ses états financiers ${ }^{13}$ ? En généralisant cette pratique, il serait alors possible de déceler hâtivement d'éventuelles difficultés financières et d'apporter promptement les correctifs nécessaires à la survie d'organisations qui, autrement, pourraient être injustement euthanasiées.

\section{Notes}

1 Horologium et mercatus sont deux mots de la langue latine qui signifient respectivement horloge et marché. On pourrait donc traduire horologium mercatus par « horloge du marché ». Quant à l'expression latine « bene diagnoscitur, bene curatur », elle se traduirait par « bien diagnostiquer, c'est bien soigner ». Il s'agit en fait d'un proverbe latin tiré du site Internet de Wikipédia, une encyclopédie en ligne dont l'adresse du portail principal est: http://fr. wikipedia.org/wiki/Accueil. L'adresse spécifique où l'on peut retrouver notre proverbe est : http://fr.wikipedia.org/ wiki/Proverbe latin.

2 En effet, selon Statistique Canada, le nombre de faillites commerciales pour les années 1998, 1999, 2000 et 2001 se chiffrait respectivement à $10791,10026,10055$ et 10405 .

3 Un peu comme une boule de cristal, Horologium mercatus est le reflet de la santé future de l'organisation. Son utilisation nécessite donc un important travail de prévision et, surtout, une ferme conviction qu'elles se réaliseront.

4 Bien sûr, nous parlons des principes comptables généralement reconnus (PCGR).

5 Bonam et mercatus sont deux mots latins qui se traduisent respectivement par bilan et marché. Bonam mercatus serait donc un bilan dont tous les éléments sont exprimés en valeur marchande.

6 La VMA correspond à la valeur actualisée nette de l'organisation. La VMA se calcule comme ceci :

$V M A=\sum_{t=1}^{n}\left(C F_{t} /(1+K)^{t}\right)-V M T$

Dans cette équation, les variables $\boldsymbol{C F}_{\boldsymbol{t}}, \boldsymbol{K}, \boldsymbol{V M T}$ et $\boldsymbol{n}$ représentent respectivement, les cash-flows d'exploitation annuels nets anticipés, le taux de rendement espéré sur les investissements (le coût du capital), la valeur marchande des actifs tangibles et le nombre d'années pendant lesquelles l'organisation espère pouvoir continuer son exploitation. Pour une explication détaillée de la VAN, lire : G. Charest, P. Lusztig et B. Schwab (1990), Gestion financière, ERPI, chapitre 5. Quant au terme cash-flow, il fait maintenant partie de la langue française. Il est égal aux recettes d'exploitation moins les déboursés d'exploitation et moins les impôts (voir le Petit Larousse ou encore consulter l'adresse Internet suivante : http://213.161.194.55/ mediadicotv5/asp/dicoweb.asp?NBD=1).

$7 \quad$ Il s'agit d'une valeur prévue puisque la VEN est largement tributaire de la valeur marchande de l'achalandage qui se calcule à partir de cash-flows prévisionnels.

8 Il ne s'agit pas ici d'une liquidation forcée mais ordonnée. On suppose en effet que l'entreprise prendra le temps de liquider ses actifs à un prix correspondant à leur valeur marchande.

9 Expression latine qui signifie à l'article de la mort. 
10 Notons bien que le deuxième et le quatrième quadrants sont divisés en deux parties égales par la diagonale située entre $4 \mathrm{~h} 30$ et $10 \mathrm{~h} 30$. Sur cette droite, VEN est toujours égale à zéro puisque VL et VMA s'annulent.

11 La théorie de l'agence prédit le comportement des actionnaires en situation de détresse financière. Pour une bonne explication de cette théorie, lire: R. Cobbaut (1987), Théorie financière, Paris, Economica, chapitre 15.
12 Notre Horologium mercatus est l'adaptation d'un modèle exposé en 1985 par le professeur Jean Roy dans un cours intitulé «Institutions financières et marché des capitaux » du programme de MBA de l'Université Laval de Québec.

13 On pourrait l'ajouter en annexe ou encore aux notes aux états financiers, avec toutes les réserves nécessaires évidemment. 


\section{PUBLICITÉ}

Vous désirez recevoir un de nos numéros déjà parus ? 